\title{
Embolismo por cristales de colesterol secundario a trombolisis: a propósito de un caso.
}

\author{
A case of embolism caused by cholesterol crystals \\ secondary to thrombolysis.
}

P. Martínez-García1, M. Salguero Villadiego² y M.L. Gutiérrez A mares1

\section{RESUMEN}

Tradicionalmente, la Medicina Forense ha tenido que dilucidar las causas del óbito en algunas muertes naturales; en particular, aquellos casos en que el fallecimiento ocurría de forma súbita o repentina y el sujeto, real o aparentemente, gozaba de buena salud. Desde la década de los 80 , el número de muertes naturales autopsiadas en el curso de Diligencias Judiciales aumenta de forma paulatina, a expensas de las defunciones que ocurren a consecuencia de complicaciones de tratamientos aplicados y posibles negligencias médicas.

El síndrome de embolismo por cristales de colesterol, es una entidad poco frecuente, que aparece principalmente en varones mayores de $\mathbf{6 0}$ años con arteriosclerosis generalizada, tras la realización de procedimientos angiográficos, cirugía vascular, o más raramente, con tratamiento anticoagulante oral, heparina o fibrinolíticos. Lo inusual del caso y sus consecuencias fatales, pueden ser de difícil comprensión a un profano, hecho que induce con facilidad a denunciar una posible malpraxis.

En el presente trabajo se expone un caso estudiado por nosotros, tras denuncia judicial, detallando el cuadro clínico, examen macro y microscópico y una revisión de la literatura.

Palabras clave: Trombolisis, embolismo por cristales de colesterol, malpraxis.

\section{ABSTRACT}

Traditionally Forensic medicine has had to explain the reasons for demise in occasional natural deaths; especially those cases in which death occurred suddenly and the subject enjoyed real or apparent good health. Since the decade of the 80 s, the number of deaths autopsied in the course of Judicial Formalities has increased slowly at the expense of deaths which occur as a consequence of complications in applied treatments and possible medical negligence.

Embolism caused by cholesterol crystals is an infrecuent entity, which appears principally in men older than 60 with widespread arteriosclerosis after angiographic procedures, vascular surgery, or, varely, upon the use of anticoagulative oral treatment, heparina or thrombolytics. The rareness of suc a case and its fatal consequences may be difficult for a layman to comprehend, a fact that easily leads to a charge of possible malpraxis.

In the present work we explain a case studied by us after judicial charges, detailing the clinical picture, macro and microscopic examination and a review of the literature.

Key words: Thrombolysis, cholesterol embolization, Malpractice.

Correspondencia: Clínica Médico Forense. Edificio Juzgados. C/ los Balbos s/n . Cádiz. Tfno: 956013059,956013060 y 956013058 .

1 Médico Forense de Cádiz.

2 Facultativo del Servicio de Anatomía Patológica. INT. Sevilla. 


\section{INTRODUCCIÓN:}

La arteriosclerosis es una enfermedad de las arterias de mediano y gran calibre, caracterizada por lesiones circunscritas de pared, denominadas placas de ateroma. Las complicaciones que pueden surgir son múltiples, entre ellas señalamos: ulceración de la placa, trombosis sobre placas ulceradas, hemorragias en el seno de las mismas y rotura de una placa arteriosclerótica con desprendimiento de parte de su contenido y producción de émbolos.

La embolia por cristales de colesterol (ECC) estaría determinada por la exposición del núcleo lipídico de una placa de ateroma a la circulación sistémica y puede aparecer de forma espontánea o tras diversas actuaciones terapéuticas.

Los trombolíticos [I] fueron la primera medida eficaz orientada a recanalizar la arteria ocluida por un trombo, con lo que se consigue la reperfusión del territorio afectado en aproximadamente el 50 a $60 \%$ de los casos. Sin embargo, su uso no está exento de riesgos.

\section{CASO ACTUAL:}

El caso que nos ocupa es el de un varón de 62 años edad que sufre dolor centrotorácico, sensación de hormigueo en manos y náuseas. Una semana antes había tenido un episodio similar sin requerir asistencia facultativa. A los 45 minutos de presentarse los síntomas, es asistido en el Hospital de su zona, diagnosticándose un infarto inferior, e iniciando fibrinolisis con Streptokinasa 1.500.000 Ul. También se trató con Solinitrina, Aspirina y Zantac, remitiéndose posteriormente al Hospital Comarcal al no haber camas disponibles en la Unidad de Coronarias.

A la media hora de su ingreso en el Hospital Comarcal (sobre las 4,30 horas después de aparecer la sintomatología), comienza con dolor en ambos miembros inferiores, acompañado de palidez intensa y ausencia de pulso. Se realiza Tomografía Axial Computerizada (TAC), que descarta disección aórtica y ante la sospecha de embolismo, se remite al Hospital Provincial.

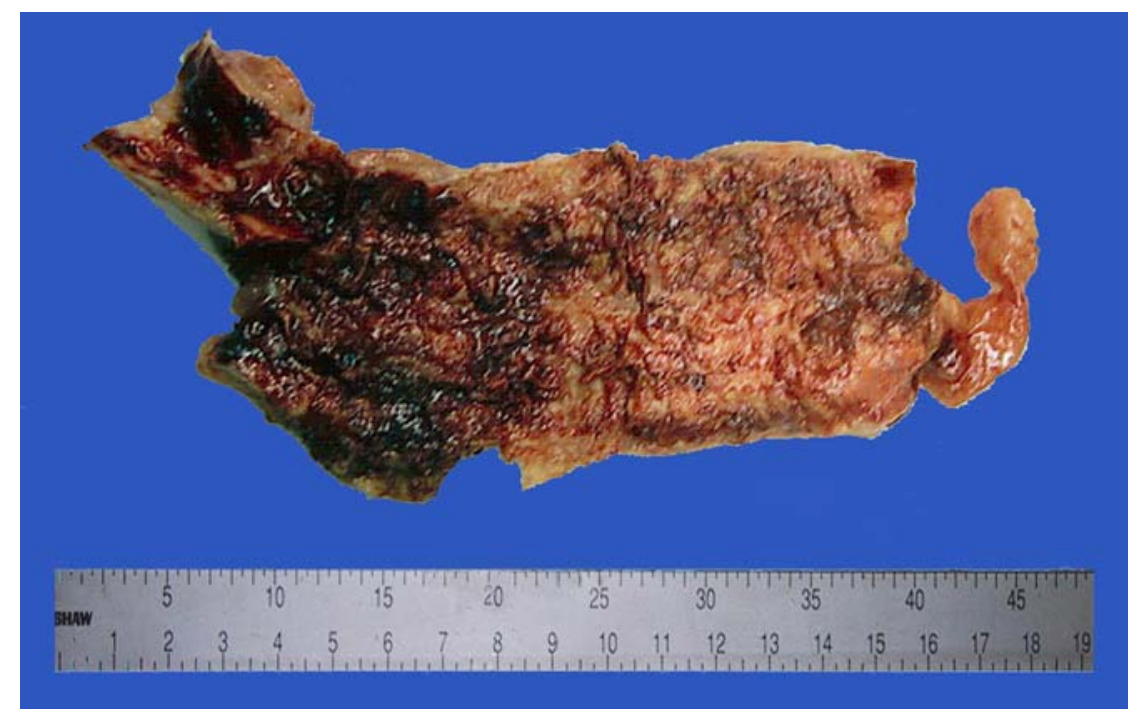

Foto 1.- Sección longitudinal de aorta abdominal con arteriosclerosis severa y material hemático coagulado. 
En el Centro de Referencia, se realiza TAC de Tórax que no muestra patología en aorta torácica y ecografía de miembros inferiores, en la que se advierte oclusión bilateral a nivel popliteo. Es intervenido, realizándose embolectomía bilateral, recuperándose la coloración, pero persistiendo la frialdad. El estudio histopatológico muestra material trombótico fibrohemático.

Pasadas las primeras horas el paciente muestra inestabilidad hemodinámica, con aparición de livideces en miembros inferiores, insuficiencia renal y shock, ocurriendo finalmente la muerte a las 24 horas de comenzar la sintomatología.

En el examen externo del cadáver se aprecia coloración rojo violada en tercio inferior de muslos, rodillas, zona genital y porción inferior del abdomen.

Los pulmones estaban aumentados de tamaño y consistencia, a la presión se percibe crepitación, permanece la huella del dedo tras la presión (Fóvea) y al corte, tras comprimir, mana espuma. El pulmón derecho pesaba 850 gramos y el izquierdo pesaba $640 \mathrm{~g}$.

Tras incidir el saco pericárdico, se observó una cardiomegalia ligera con un peso de 390 gramos. El ventrículo izquierdo estaba hipertrófico con un grosor máximo en su pared libre de $2 \mathrm{~cm}$. El análisis histopatológico del corazón muestra una cardiopatía isquémica crónica, sobre la que asienta un infarto antiguo y otro reciente, de I a 3 semanas de evolución, en la cara posterior del ventrículo izquierdo.

La aorta torácica presenta una gran placa arterioesclerótica rota, con una hemorragia intraplaca y material trombótico adherido por debajo del cayado (fotos I y 2). El endotelio de la aorta abdominal está ocupado por material hemático coagulado de coloración rojiza. El hígado exhibía aspecto esteatósico. El examen del hígado y pulmón confirma los diagnósticos macroscópicos de esteatosis y edema pulmonar.

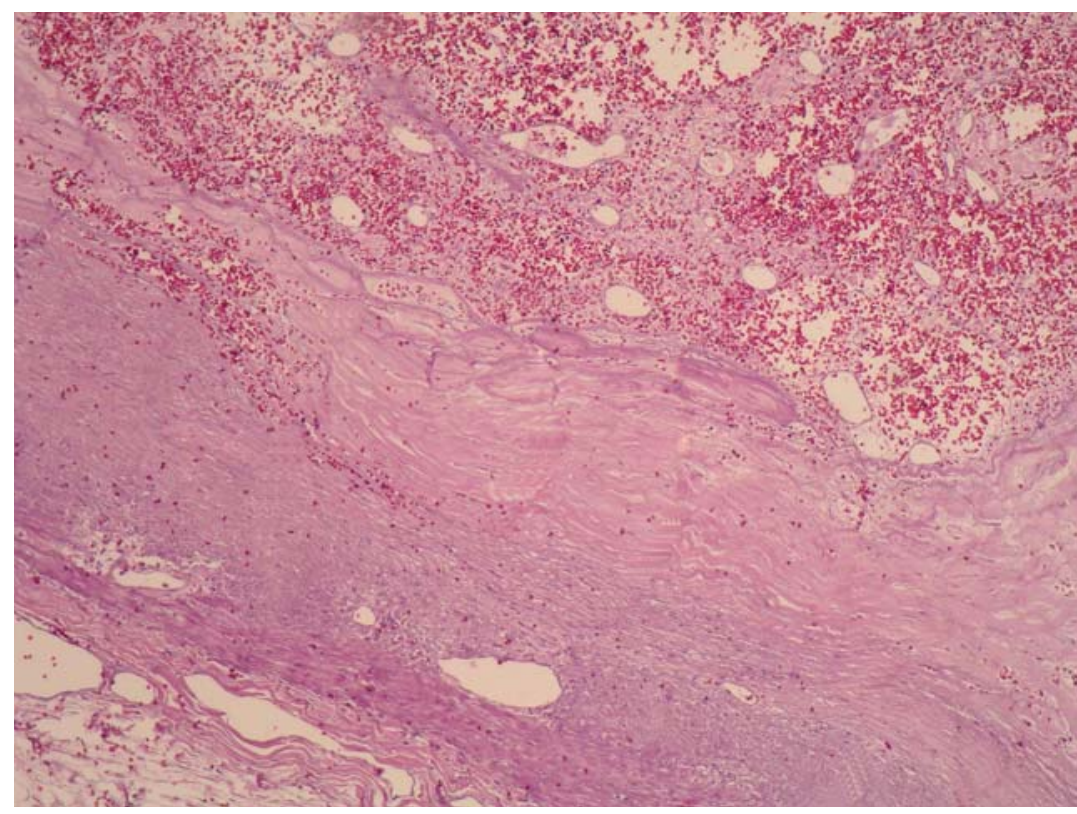

Foto 2.- Placa arterioesclerótica rota con una hemorragia intraplaca. 
En las arteriolas encargadas de irrigar la musculatura esquelética de las piernas, se advierten numerosos émbolos procedentes de placas de ateroma rotas que producen necrosis isquémica de las fibras musculares (foto 3 ).

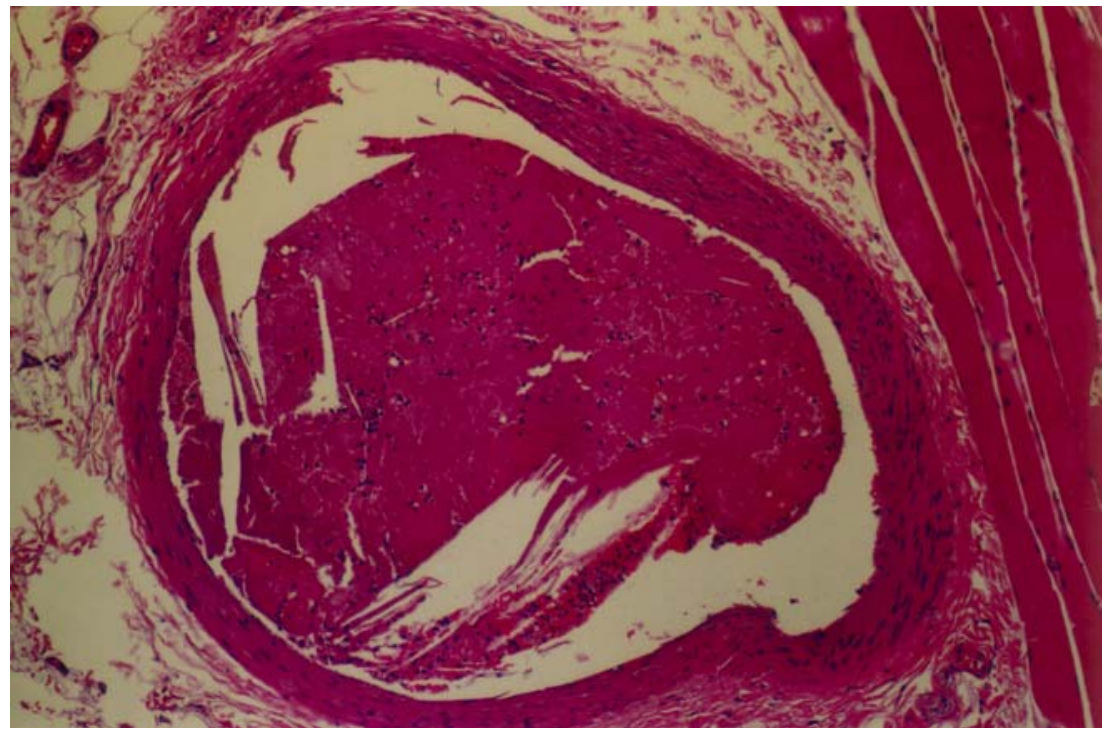

Foto 3.- Arteriolas encargadas de irrigar la musculatura esquelética con numerosos émbolos procedentes de placas de ateromas rotas.

Histológicamente los riñones presentan necrosis tubular aguda, pigmento mioglobínico en túbulos y émbolos en arteriolas procedentes de la rotura de placas de ateroma (foto 4).

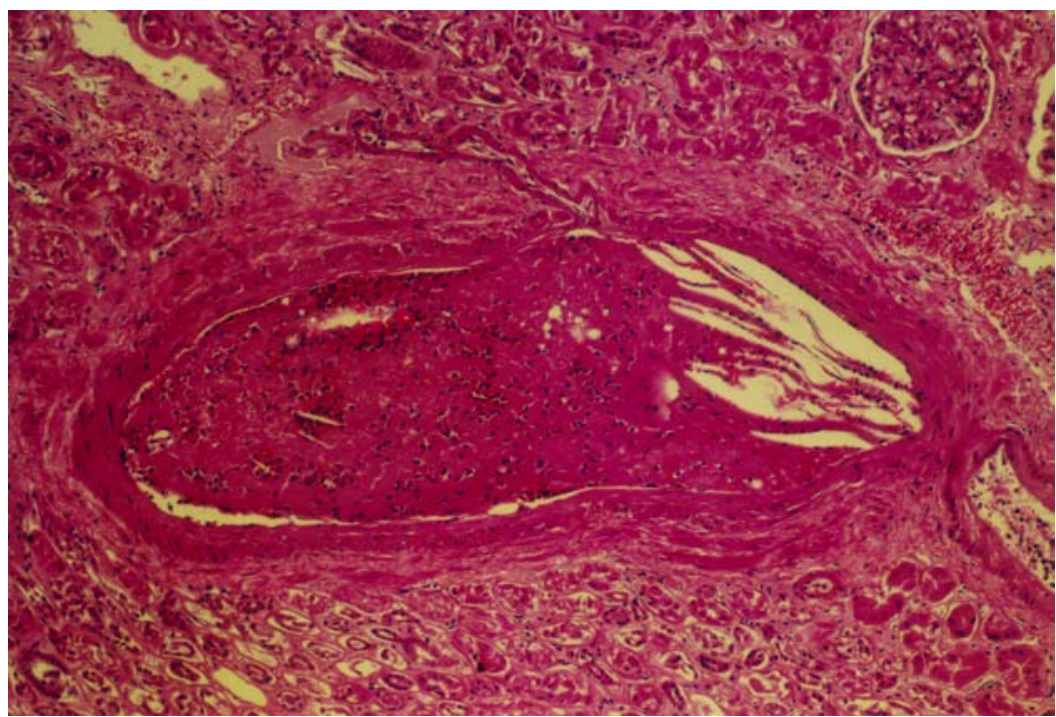

Foto 4.- Émbolos en arteriolas renales procedentes de la rotura de placas de ateroma. 


\section{DISCUSIÓN:}

La arteriosclerosis es una enfermedad de las arterias de mediano y gran calibre, caracterizada por lesiones circunscritas de pared, denominadas placas de ateroma. Estas se hallan formadas por lípidos, detritos celulares y calcio, cubiertas por una cápsula fibrosa constituida por colágeno, elastina, fibras musculares lisas, macrófagos y linfocitos, que está tapizada por células endoteliales [2]. Las placas de ateroma pueden darse a todo lo largo de la aorta y demás arterias de la economía, incluso en las de diámetro inferior a $2 \mathrm{~mm}$; pero la aorta abdominal es la más severamente afectada [3].

Es conocido [4] que la placa de ateroma está constituida por: a) una cápsula fibrosa compuesta por músculo liso, leucocitos, tejido conectivo y material de membrana basal; b) un área celular interna formada por macrófagos, CML y linfocitos; y c) un núcleo necrótico que contiene restos celulares, cristales de colesterol intra o extra celulares, calcio, etc. Con el tiempo se produce una proliferación de los vasa vasorum, que pueden llegar hasta la íntima de la arteria.

La embolia por cristales de colesterol (ECC) estaría determinada por la exposición del núcleo lipídico de una placa de ateroma a la circulación sistémica [5]. La rotura de la lesión arterioesclerótica, y el vaciamiento del contenido lipídico, puede ser secundaria a su calcificación o ser consecutiva a una hemorragia repentina en su parte central [6]. La presencia de placas de ateroma con mas de $4 \mathrm{~mm}$ de espesor, placas ulceradas o con proyecciones móviles hacia la luz vascular, se va a correlacionar directamente con fenómenos embólicos sistémicos [7]. En el caso que presentamos, una importante hemorragia aguda intraplaca fue la responsable de la rotura de la misma y la posterior embolización de numerosos fragmentos en las arteriolas del riñón y de los miembros inferiores.

La ECC, es una enfermedad rara que puede ocurrir espontáneamente, tras cirugía vascular, cateterismo cardiaco, aortografía y angioplastia coronaria [8]. En un principio se pensó que los tratamientos con trombolíticos y anticoagulantes eran simples factores coadyuvantes; sin embargo, Pochmalicki y cols [9] recogen en 1993 cuatro casos de la literatura y uno propio, en que el único factor desencadenante es la inyección de sustancias trombolíticas. Ribera [10], en una serie de 16 casos, encuentra como factor desencadenante en el $44 \%$ de las ocasiones, los tratamientos con heparina, el $31 \%$ de los mismos asociados a fibrinolíticos y el $25 \%$ a dicumarínicos.

Algunos autores [ $\mathrm{II}$ ] consideran el uso temprano de agentes trombolíticos como el tratamiento más importante del infarto de miocardio; sin embargo, no está exento de complicaciones.

La aparición de fenómenos adversos mas frecuentemente observada, en la trombolisis, han sido [12]: fracaso de lisis del coágulo, retrombosis, hemorragia local y sistémica, infarto de miocardio hemorrágico y disección arterial coronaria. Otros autores también han descrito: lisis de trombos murales [13] (que ocurriría en las áreas mas recientemente coaguladas) [14], embolismo pulmonar masivo por fragmento liberado de una trombosis venosa profunda y microinfartos de piel [15]. Es posible que el tratamiento trombolítico haya jugado un papel fundamental en la producción de la hemorragia aguda intraplaca que se advierte en la aorta torácica por debajo del cayado. Dicha hemorragia es la responsable de la posterior rotura y embolización.

El tiempo de latencia entre la administración de fibrinolíticos y la aparición de fenómenos tromboembólicos oscila entre una hora y 28 días [16]. Si ocurre a partir de la aorta abdominal o la torácica por debajo del cayado, se verían afectadas de forma preferente las arterias renales, digestivas y de miembros inferiores; en caso de que la placa rota fuera torácica las manifestaciones serían miocárdicas, oculares o neurológicas. 
Las manifestaciones clínicas son muy variadas destacando [ 17 ] las correspondientes a fracaso renal, livedo reticularis, acrocianosis, dolor abdominal y perforación intestinal, mialgias, mononeuristis y dolor torácico por IAM. También se han descrito [18] púrpura, gangrena, nódulos subcutáneos y úlceras isquémicas.

Dada la frecuencia en la que se encuentra afectación cutánea, el diagnóstico puede establecerse por biopsia cutánea [19], método poco agresivo y muy accesible[20]. El estudio histológico permite comprobar, en los vasos cutáneos, la existencia de trombos con espacios espiculares vacíos que se corresponderían con los cristales de colesterol. Según el tiempo de evolución [2I] puede objetivarse la presencia de neutrófilos, eosinófilos, células mono o multinucleares, etc.

El pronostico del cuadro es sombrío dado que el 50 \% de los pacientes afectos de ECC fallecen a consecuencia de las complicaciones renales, gástricas o cardiovasculares [22].

\section{CONCLUSIONES:}

Los avances en el tratamiento y prevención del infarto agudo de miocardio han logrado disminuir la mortalidad hospitalaria hasta un 7-10\%; sin embargo, esta entidad sigue siendo la principal causa de muerte en los países desarrollados [23]. Está perfectamente establecido que el tratamiento fibrinolítico reduce la mortalidad en pacientes con síndrome coronario agudo, observándose un descenso alrededor del $23 \%$ en la mortalidad intrahospitalaria, siendo esta reducción de hasta el 50\% en los pacientes tratados en la primera hora de evolución de los síntomas [24]. Este beneficio se debe a diversas causas, como la limitación del tamaño del infarto y la preservación de la función ventricular, principal factor pronóstico en los supervivientes de un IAM [25]

Esta técnica no está exenta de riesgos, contemplándose como complicación más importante la hemorragia cerebral. El embolismo por Cristales de Colesterol es una entidad mucho mas infrecuente, por lo que supone un "Riesgo Conocido" muy inferior al proceso isquémico en sí, lo que justifica, en nuestra opinión, sobradamente su utilización.

\section{AGRADECIMIENTO:}

Nuestro más sincero agradecimiento por su colaboración a D. Joaquín Lucena, Jefe del Servicio de Patología Forense del IML de Sevilla, y a D. Manuel Galván, Auxiliar de la Administración de Justicia del Servicio de Patología Forense del IML de Sevilla, por su inestimable ayuda en el tratamiento digital de las imágenes.

\section{BIBLIOGRAFÍA:}

I.- Prieto ] C, Corbalan R, Nazza , C et al. Cambios en los patrones de prescripción de medicamentos en el infarto agudo del miocardio.: Comparación de dos períodos. Rev. méd. Chile, mayo 200I, vol.I29, no.5, p.48I-488. ISSN 0034-9887.

2.- Jurado J y Miquel C. Arteriosclerosis. En: Rozman C. Medicina Interna Farreras-Rozman. 13 edición. Mosby Doyma Libros. Barcelona, 1996. Pp 637-642

3.- Anderson J R. Vasos Sanguíneos y linfáticos. En: Anderson J R. Patología de Muir. Edit Espax SA. Barcelona 1979. Pp 363-398.

4.- Pardo F J. Anatomía Patológica de los Vasos. En: Pardo F J. Anatomía Patológica. Edit Harcourt. Madrid. 2000.Pp527-557.

5.- Gallego JL y Cols. Lesiones cutáneas e insuficiencia renal tras infarto Aguido de Miocardio. Rev Esp Cardiol. 2001;54:13391342.

6.- Haust M D. Arteriosclerosis. En: Brunson J G y Gall E A. Tratado de Patología Humana. Edit Interamericana. Mexico 1975. Pp 438-472.

7.- Barbón J J y Cols. Embolismo retiniano por placa de ateroma en arco aortico. Arch Soc Esp Oftalmol 200I; 76:735-738.

8.- López F y Cols. Embolismo de colesterol durante el tratamiento con heparina. Rev Esp Cardiol. 1993; 46: 755-757

9.- Pochmalicki $G$ y cols. Embolies diffuses de Cholesterol aprés Fibrinolyse pour Infarctuse Myocarde. Arch Mal Coeur 1993; 86: 263-266.

10.- Ribera M y Cols. Enfermedad por Émbolos de Colesterol: Estudio de 16 casos. Rev Clin Esp 2000; 44 (I2): 659-663.

II.- Srimahacholota $S$ y Cols. Efficacy of Rapid Infusion of Streptokinase in patients With Acute Miocardial Infarction. J Med Assoc Thai 2000; 83 (I): 8-I2. 
12.- Menke DM y Cols. Histologic Evidence Of Distal Coronary Thromboembolism. A Complication Of Acute Proximal Coronary Artery Thrombolysis Therapy. Chest. 1986; 90 (4): 614-6.

13.- Zahger D y cols. Systemic Embolization Following Thrombolytic Therapy For Acute Myocardial Infarction. Chest 1990; 97: 754-756. 14.- Travis VD y Balogh K. Saddle Embolism Of The Aorta. A complication Of Streptokinase Therapy. Cardiology. 1986; 73: 156-159. 15.- Abrahan JS y Cols. Microembolism Fron Aortic aneurysm And Ventricular Thrombus: A Complication of Intravenous Streptokinase. Postgrad Med J. 1994; 70: 756-8.

16.- Santamaría A y cols. Embolismo por Cristales de Colesterol: A Proposito de un caso. An. Med. Interna. 2001;18 (4):201-204.

17.- Ena J y Cols. Isquemia Digital, Insuficiencia Renal y Livedo Reticularis tras realizar fibrinolisis. Rev Clin Esp. 1996; 196 (3): 59-60. 18.- Bolognia JL y Braverman IM. Manifestaciones cutáneas de Enfermedades Internas. EN: Fauci AS y cols. Principios de Medicina Interna Harrisson. I4 edición. Vol I. Edit McGraw-Hill Interamericana. Madrid. 2000. Pp 354-372.

19.- Hawthorn IE y cols. Severe Lower Limb Ischaemia Vit Pulses: Cholesterol Embolisation- a Little Known Complication Of Aortic Surgery. Eur J Vasc Surg. 1993; 7 (4): 470-4.
20.- Ostabal M y Cols. Livedo Reticular y Hemoptisis Como Forma de Presentación de un Caso de Embolismos Múltiples de Colesterol. An Med Interna. 1997; I4 (I2): 651.

21.- Sanchez J y Camacho F. Aspectos etiopatogénicos y diagn 'sticos de los embolismos por colesterol. Rev Clin Esp. 2000; 200 (I2): 647-8.

22.- Suarez R Y cols. "La biopsia cutánea en el Diagnóstico de Patología Sistémica por microembolos de Colesterol. An Med Interna. 1999; 15 (6): 321.

23.- García Gil D. y col: Diagnóstico Tratamiento Inicial del Infarto Agudo de Miocardio no complicado en Urgencias. En: García Gil D y col. Manual de Urgencias. Edit Daniel García Gil. Madrid 2000. Pp 122-129.

24.- Sitges M, Bosch X y Betriu A. Optimización del tratamiento trombolítico en el infarto agudo de miocardio: papel de los nuevos fármacos fibrinoselectivos y de la asociación con los nuevos antitrombóticos. Rev Esp Cardiol 1998; 51: 178-191.

25.- Sitges M y Col. Mejoría del tratamiento del infarto agudo de miocardio mediante la creación de una unidad de trombólisis en el servicio de cardiología. Rev Esp Cardiol 1998; 51: 732-739. 